\title{
Convergence Analysis of a Discontinuous Finite Volume Method for the Signorini Problem
}

\author{
Yuping Zeng ${ }^{1} \&$ Fen Liang $^{1}$ \\ ${ }^{1}$ School of Mathematics, Jiaying University, Meizhou 514015, China \\ Correspondence: Yuping Zeng, School of Mathematics, Jiaying University, Meizhou 514015, China. \\ E-mail: zeng_yuping@163.com
}

Received: June 1, 2020 Accepted: June 23, 2020 Online Published: July 10, 2020

doi:10.5539/jmr.v12n4p49 URL: https://doi.org/10.5539/jmr.v12n4p49

\begin{abstract}
We introduce and analyze a discontinuous finite volume method for the Signorini problem. Under suitable regularity assumptions on the exact solution, we derive an optimal a priori error estimate in the energy norm.
\end{abstract}

Keywords: signorini problem, discontinuous finite volume method, a priori error estimate

AMS subject classifications: $65 \mathrm{~N} 15 ; 65 \mathrm{~N} 30 ; 49 \mathrm{~J} 40$

\section{Introduction}

The Signorini problem belongs to a variational inequality of the first kind, and it is used to describe the unilateral contact model. The theory of variational inequalities has been made applications in various fields such as mechanics, physics, and financial engineering. The detailed mathematical analysis of variational inequalities can be found in the monograph Kinderlehrer \& Stampacchia (1980). In the context of numerical methods for such problems, finite element (FE) methods are commonly used approaches, and they have been studied extensively and deeply. For instance, we refer the reader to Falk (1974), Brezzi et al. (1977), Scarpini \& Vivaldi (1977), Glowinski (2008), Ben Belgacem (2000), Chen \& Nochetto (2000), Veeser (2001), Hild \& Nicaise (2005), Carstensen \& Hu (2015) for conforming FE methods, and to Wang (2003), Hua \& Wang (2007), Shi et al. (2012), Shi \& Xu (2013), Carstensen \& Köhler (2017) for nonconforming FE methods. In addition, discontinuous Galerkin (DG) (cf. Arnold et al., 2002) method is also an effective numerical scheme for solving variational inequalities, and it has made tremendous progress in the past decade, please see Djoko (2008), Wang et al. (2010, 2011), Bustinza \& Sayas (2012), Gudi \& Kamana (2014, 2016), Zeng et al. (2015).

Compared with the rich results of the FE method, the finite volume (FV) method for solving variational inequalities is still very rare. The main idea of FV methods is to integrate the partial differential equations in a control volume, and thus they satisfy some conservation properties. We refer the reader to the monographs Eymard et al. (2000) and Li et al. (2000), and to the articles Cai et al. (1991), Huang \& Xi (1998), Ewing et al. (2002), Wu \& Li (2003), Chou \& Ye (2007), Xu \& Zou (2009), Chen (2010), Yang \& Liu (2010), Lv \& Li (2012), Zhang \& Zou (2015), Guo et al. (2019), Zhang et al. (2019) (see also the references therein) for detailed presentation of such methods. More recently, Zhang \& Tang (2015) have investigated a conforming FV method to solve two kinds of variational inequalities, including the obstacle and simplified frictional problems. Later, Zhang \& Li (2015) also extended this method to the Signorini problem and established a super-close interpolation estimate. The goal of this article is to design discontinuous finite volume (DFV) methods for solving the Signorini problem. DFV methods were initially proposed and analyzed by Ye (2004) to address the second order problems. Inheriting attractive properties of both DG and FV methods, DFV methods can easily handle highly nonuniform meshes and inhomogeneous boundary conditions. In addition, DFV methods have the localizability of discontinuous elements and the corresponding dual partitions that make them suitable for parallel computation. Moreover, compared with classical conforming and nonconforming FV methods, DFV methods have small support in the dual elements. For these reasons, DFV methods have been used to solve second order elliptic equations (Kumar et al., 2009; Bi \& Ge, 2012; Liu et al., 2012; Carstensen et al., 2016), Stokes equations (Ye, 2006; Cui \& Ye, 2010; Kumar \& Ruiz-Baier, 2015; Wang et al., 2018; Carstensen et al., 2018), Darcy-Stokes problems (Wang et al., 2016; Li et al., 2018), Biot equations (Kumar et al., 2020), phase field model (Li et al., 2020) and other problems (Bürger et al., 2015; Kumar et al., 2019). In the present work, we aim at developing the DFV method for the Signorini model. To carry out a priori error analysis, we shall deal with two main difficulties that come from the nonlinearity of the Signorini problem and the complexity of bilinear form of DFV methods.

The article is organized as follows. We state the model problem and its DFV scheme in Section 2. A detailed error estimate in the mesh-dependent norm is established in Section 3. Finally, in Section 4, we make some conclusions. 


\section{The DFV Method for the Signorini Problem}

\subsection{Signorini Problem and Its Weak Formulation}

Let $\Omega \subset \mathbb{R}^{2}$ be a convex bounded polygonal domain with boundary $\partial \Omega$, given $f \in L^{2}(\Omega)$, we are concerned with the following Signorini problem:

$$
\begin{aligned}
-\Delta u & =f & & \text { in } \Omega, \\
u & =0 & & \text { on } \Gamma_{D}, \\
\frac{\partial u}{\partial \mathbf{n}} & =0 & & \text { on } \Gamma_{N}, \\
u \geq 0, \frac{\partial u}{\partial \mathbf{n}} \geq 0, & u \frac{\partial u}{\partial \mathbf{n}}=0 & & \text { on } \Gamma_{C},
\end{aligned}
$$

where $\frac{\partial u}{\partial \mathbf{n}}=\nabla u \cdot \mathbf{n}$, with $\mathbf{n}$ being the unit exterior normal vector, and $\Gamma_{D}, \Gamma_{N}$ and $\Gamma_{C}$ are three disjoint parts of $\partial \Omega$ with $\left|\Gamma_{D}\right|>0$ and $\left|\Gamma_{C}\right|>0$.

For $\mathcal{D} \in \mathbb{R}^{2}$, we write $H^{m}(\mathcal{D})$ to stand for the usual Sobolev space with regularity exponent $m \geq 0$. Its norm and seminorm are denoted by $\|\cdot\|_{m, \mathcal{D}}$ and $|\cdot|_{m, \mathcal{D}}$, respectively. When $\mathcal{D}=\Omega$, we simply write $\|\cdot\|_{m, \Omega}\left(\operatorname{resp} .|\cdot|_{m, \Omega}\right)$ by $\|\cdot\|_{m}\left(\operatorname{resp} .|\cdot|_{m}\right)$. If $m=0, H^{0}(\mathcal{D})$ can be understood as $L^{2}(\mathcal{D})$. Let

$$
H_{\Gamma_{D}}^{1}(\Omega)=\left\{v \in H^{1}(\Omega): v=0 \text { on } \Gamma_{D}\right\},
$$

and

$$
K=\left\{v \in H_{\Gamma_{D}}^{1}(\Omega): v \geq 0 \text { on } \Gamma_{C}\right\}
$$

The weak form of the problem (1) reads: Seek $u \in K$ satisfying

$$
\int_{\Omega} \nabla u \cdot \nabla(v-u) d x \geq \int_{\Omega} f(v-u) d x \forall v \in K .
$$

\subsection{DFV Method}

Denote by $\mathcal{T}_{h}$ a shape-regular triangulation of $\Omega$ into triangular elements $\{T\}$, and it is referred to the primal mesh. Let $h_{T}=\operatorname{diam}(T)$ and $h=\max _{T \in \mathcal{T}_{h}} h_{T}$. The set of interior edges is denoted by $\mathcal{E}_{h}^{I}$. Similarly, we use $\mathcal{E}_{h}^{D}, \mathcal{E}_{h}^{N}$, and $\mathcal{E}_{h}^{C}$ to denote the sets of edges on $\Gamma_{D}, \Gamma_{N}$ and $\Gamma_{C}$, respectively. As a result, the set of all edges can be written as $\mathcal{E}_{h}=\mathcal{E}_{h}^{I} \cup \mathcal{E}_{h}^{D} \cup \mathcal{E}_{h}^{N} \cup \mathcal{E}_{h}^{C}$. Moreover, each edge $e \in \mathcal{E}_{h}$ is fixed with an unit normal $\mathbf{n}$, in the sense that on the boundary edge, $\mathbf{n}$ stands for the exterior unit normal. In addition, the dual mesh $\mathcal{T}_{h}^{*}$ of $\mathcal{T}_{h}$ is constructed as follows. For each element $T$, we divide it by connecting its vertices and barycenter, see Fig.1. In what follows, all generic constants (with or without subscripts) in this article are independent of $h$, but depend on the minimum angle of elements.

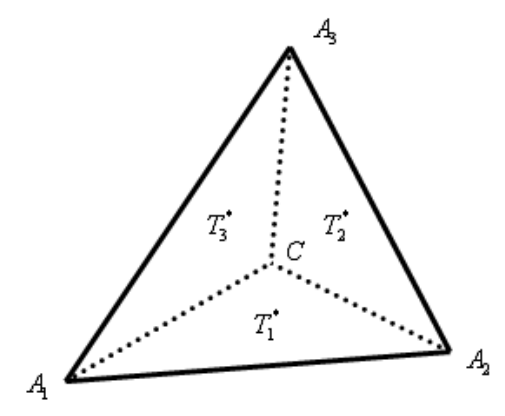

(a)

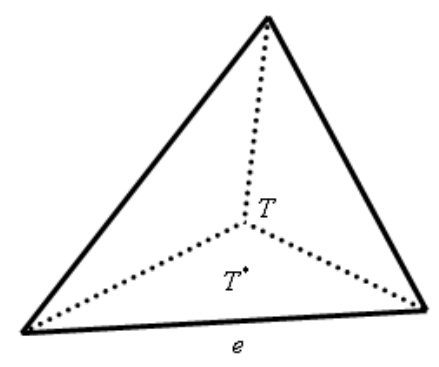

(b)

Figure 1. A primal mesh and its dual volume for DFV method

For $e \in \mathcal{E}_{h}^{I}$ satisfying $e=T^{+} \cap T^{-}$, consider a discontinuous function $v$, we define its average and jump by

$$
\{v\}=\frac{1}{2}\left(\left.v\right|_{T^{+}}+\left.v\right|_{T^{-}}\right) \text {and } \llbracket v \rrbracket=\left.v\right|_{T^{+}}-\left.v\right|_{T^{-}} .
$$


On a boundary edge, we set

$$
\{v\}=v \text { and } \llbracket v \rrbracket=v .
$$

For trial functions corresponding to $\mathcal{T}_{h}$, we consider the discontinuous linear element space:

$$
V_{h}=\left\{v \in L^{2}(\Omega):\left.v\right|_{T} \in \mathbb{P}_{1}(T) \quad \forall T \in \mathcal{T}_{h}\right\} .
$$

Here and in the following, $\mathbb{P}_{k}(T)$ is the space of polynomials of degree $\leq k$ on $T$. Moreover, we approximate the set $K$ by the following convex subset of $V_{h}$ (cf. Wang et al., 2011):

$$
K_{h}=\left\{v_{h} \in V_{h}: v_{h}(x) \geq 0 \text { at all nodes on } \overline{\Gamma_{C}}\right\} .
$$

Since the linear finite element is used, it is easy to check that $v_{h} \geq 0$ at all nodes on $\overline{\Gamma_{C}}$ implies that $v_{h} \geq 0$ on $\overline{\Gamma_{C}}$.

We also introduce the test space with regard to the dual mesh $\mathcal{T}_{h}^{*}$ :

$$
V_{h}^{*}=\left\{v \in L^{2}(\Omega):\left.v\right|_{T^{*}} \in \mathbb{P}_{0}\left(T^{*}\right) \forall T^{*} \in \mathcal{T}_{h}^{*}\right\} .
$$

Let $V(h)=V_{h}+\left[H_{\Gamma_{D}}^{1}(\Omega) \cap H^{2}(\Omega)\right]$, we define an operator $\gamma_{h}: V(h) \rightarrow V_{h}^{*}$ by

$$
\left.\gamma_{h} v\right|_{T^{*}}=\left.\frac{1}{h_{e}} \int_{e} v\right|_{T^{*}} d s \forall T^{*} \in \mathcal{T}_{h}^{*},
$$

where $h_{e}$ denotes the length of the edge $e$ (see Fig.1).

Inspired by Ye (2004), we define the bilinear form of the DFV method:

$$
\begin{aligned}
A(w, v)= & A_{1}(w, v)-\sum_{e \in \mathcal{E}_{h}^{I} \cup \mathcal{E}_{h}^{D}} \int_{e}\left\{\frac{\partial w}{\partial \mathbf{n}}\right\} \llbracket \gamma_{h} v \rrbracket d s-\sum_{e \in \mathcal{E}_{h}^{I} \cup \mathcal{E}_{h}^{D}} \int_{e}\left\{\frac{\partial v}{\partial \mathbf{n}}\right\} \llbracket \gamma_{h} w \rrbracket d s \\
& +\sum_{e \in \mathcal{E}_{h}^{I} \cup \mathcal{E}_{h}^{D}} \beta_{e} h_{e}^{-1} \int_{e} \llbracket \gamma_{h} w \rrbracket \llbracket \llbracket \gamma_{h} v \rrbracket d s,
\end{aligned}
$$

with $A_{1}(w, v)=-\sum_{T^{*} \in \mathcal{T}_{h}^{*}} \int_{\partial T^{*}}\left(\frac{\partial w}{\partial \mathbf{n}}\right) \gamma_{h} v d s+\sum_{T \in \mathcal{T}_{h}} \int_{\partial T}\left(\frac{\partial w}{\partial \mathbf{n}}\right) \gamma_{h} v d s$. Similar to the symmetric interior penalty DG method, $\beta_{e}$ is called the penalty parameter. We need to choose large enough $\beta_{e}$ to satisfy the coercivity (see the inequality (7) below).

Now, we state the DFV discrete scheme for the problem (1): Find $u_{h} \in K_{h}$ satisfying

$$
A\left(u_{h}, v_{h}-u_{h}\right) \geq \int_{\Omega} f\left(\gamma_{h} v_{h}-\gamma_{h} u_{h}\right) d x \quad \forall v_{h} \in K_{h} .
$$

\section{Error Estimates}

As in Ye (2004), we define the mesh-dependent norm on $V(h)$ :

$$
\|v\|_{h}=\left(\sum_{T \in \mathcal{T}_{h}}\|\nabla v\|_{0, T}^{2}+\sum_{e \in \mathcal{E}_{h}^{L} \cup \mathcal{E}_{h}^{D}} \llbracket \gamma_{h} v \|^{2}+\sum_{T \in \mathcal{T}_{h}} h_{T}^{2}|v|_{2, T}^{2}\right)^{1 / 2} .
$$

We then have the following properties in relation to $A(\cdot, \cdot)$, more details please see Lemmas 2.2 and 2.3 in Ye (2004).

Lemma 3.1. There holds

$$
|A(w, v)| \leq C_{a}\|w\|_{h}\|v\|_{h} \quad \forall w, v \in V(h) .
$$

If $\beta_{e}$ is large enough, it holds that

$$
A(v, v) \geq C_{b}\|v\|_{h}^{2} \quad \forall v \in V_{h}
$$

We recall the following results that are useful in the forthcoming analysis (cf. Chou \& Ye, 2007).

\section{Lemma 3.2.}

$$
\begin{aligned}
& \text { if } \llbracket v \rrbracket=0, \text { then } \llbracket \gamma_{h} v \rrbracket=0 . \\
& \left\|v-\gamma_{h} v\right\|_{0, e} \leq C h_{T}^{1 / 2}\|\nabla v\|_{0, T} \quad \forall v \in V(h),
\end{aligned}
$$

where $e \in \mathcal{E}_{h}^{T}$, with $\mathcal{E}_{h}^{T}=\left\{e \in \mathcal{E}_{h}: e \subset \partial T\right\}$. 
In addition, let $u_{I}$ be the continuous linear interpolant of $u$, it satisfies (cf. Brenner \& Scott, 2008; Wang \& Shi, 2010):

$$
\left\|u-u_{I}\right\|_{m, T} \leq C h^{2-m}|u|_{2, T}, \quad m=0,1,2 .
$$

Note that $\llbracket u \rrbracket=0\left(\forall e \in \mathcal{E}_{h}^{I} \cup \mathcal{E}_{h}^{D}\right)$, it follows from (8) that $\llbracket \gamma_{h} u \rrbracket=0\left(\forall e \in \mathcal{E}_{h}^{I} \cup \mathcal{E}_{h}^{D}\right)$. Thus,

$$
\left\|u-u_{I}\right\|_{h}=\left(\sum_{T \in \mathcal{T}_{h}}\left(\left\|\nabla\left(u-u_{I}\right)\right\|_{0, T}^{2}+h_{T}^{2}\left|u-u_{I}\right|_{2, T}^{2}\right)\right)^{1 / 2} .
$$

This together with (10) yields

$$
\left\|u-u_{I}\right\|_{h} \leq C h|u|_{2} .
$$

We recall the trace inequality (see e.g., Arnold et al., 2002):

$$
\|w\|_{0, e}^{2} \leq C\left(h_{e}^{-1}\|w\|_{0, T}^{2}+h_{e}\|\nabla w\|_{0, T}^{2}\right) \forall w \in H^{1}(T)
$$

where $e \in \mathcal{E}_{h}^{T}$. It is necessary to point out that, the constant $C$ in (9), (10) and (13) is not the same.

Following (Brezzi et al., 1977; Zeng et al., 2015), we provide an optimal order error estimate in the energy norm defined in (5).

Theorem 3.3. Let $u$ and $u_{h}$ be the solutions of (1) and (4), respectively. Assume that $u \in H^{2}(\Omega), \frac{\partial u}{\partial \mathbf{n}} \mid \Gamma_{C} \in L^{\infty}\left(\Gamma_{C}\right)$, and the number of transition points between contact and noncontact is finite, there holds

$$
\left\|u-u_{h}\right\|_{h} \leq C h\left(\|u\|_{2}+\left\|\frac{\partial u}{\partial \mathbf{n}}\right\|_{L^{\infty}\left(\Gamma_{C}\right)}\right) .
$$

Proof. The triangle inequality gives

$$
\left\|u-u_{h}\right\|_{h} \leq\left\|u-u_{I}\right\|_{h}+\left\|u_{I}-u_{h}\right\|_{h} .
$$

Note that the bound of $\left\|u-u_{I}\right\|_{h}$ is stated in (12), it remains to estimate the second term $\left\|u_{I}-u_{h}\right\|_{h}$. From (7), we have

$$
C_{b}\left\|u_{I}-u_{h}\right\|_{h}^{2} \leq A\left(u_{I}-u_{h}, u_{I}-u_{h}\right) \equiv \mathbb{D}_{1}+\mathbb{D}_{2},
$$

where

$$
\begin{aligned}
& \mathbb{D}_{1}=A\left(u_{I}-u, u_{I}-u_{h}\right), \\
& \mathbb{D}_{2}=A\left(u-u_{h}, u_{I}-u_{h}\right) .
\end{aligned}
$$

For the first term $\mathbb{D}_{1}$, we infer from (6) and Young's inequality that

$$
\mathbb{D}_{1} \leq C_{a}\left\|u_{I}-u\right\|_{h}\left\|u_{I}-u_{h}\right\|_{h} \leq \frac{C_{a}}{4 \epsilon_{1}}\left\|u_{I}-u\right\|_{h}^{2}+C_{a} \epsilon_{1}\left\|u_{I}-u_{h}\right\|_{h}^{2}
$$

Next, we focus on estimating the second term $\mathbb{D}_{2}$. Since $\llbracket u \rrbracket=0\left(\forall e \in \mathcal{E}_{h}^{I} \cup \mathcal{E}_{h}^{D}\right)$, this together with (8) implies that $\llbracket \gamma_{h} u \rrbracket=0\left(\forall e \in \mathcal{E}_{h}^{I} \cup \mathcal{E}_{h}^{D}\right)$. Moreover, note that $\llbracket \frac{\partial u}{\partial \mathbf{n}} \rrbracket=0\left(\forall e \in \mathcal{E}_{h}^{I}\right), \frac{\partial u}{\partial \mathbf{n}}=0$ on $\Gamma_{N}$ and $V_{h}^{*}$ is piecewise constant space, we apply integrating by parts to find that

$$
\begin{aligned}
& A\left(u, u_{I}-u_{h}\right) \\
= & -\sum_{T^{*} \in \mathcal{T}_{h}^{*}} \int_{\partial T^{*}}\left(\frac{\partial u}{\partial \mathbf{n}}\right) \gamma_{h}\left(u_{I}-u_{h}\right) d s+\sum_{T \in \mathcal{T}_{h}} \int_{\partial T}\left(\frac{\partial u}{\partial \mathbf{n}}\right) \gamma_{h}\left(u_{I}-u_{h}\right) d s \\
& -\sum_{e \in \mathcal{E}_{h}^{I} \cup \mathcal{E}_{h}^{D}} \int_{e}\left\{\frac{\partial u}{\partial \mathbf{n}}\right\} \llbracket \gamma_{h}\left(u_{I}-u_{h}\right) \rrbracket d s \\
= & \sum_{T^{*} \in \mathcal{T}_{h}^{*}} \int_{T^{*}}-\Delta u \gamma_{h}\left(u_{I}-u_{h}\right) d x+\sum_{e \in \mathcal{E}_{h}} \int_{e}\left\{\frac{\partial u}{\partial \mathbf{n}}\right\} \llbracket\left[\gamma_{h}\left(u_{I}-u_{h}\right) \rrbracket d s\right. \\
& +\sum_{e \in \mathcal{E}_{h}^{I}} \int_{e} \llbracket \frac{\partial u}{\partial \mathbf{n}} \rrbracket\left\{\gamma_{h}\left(u_{I}-u_{h}\right)\right\} d s-\sum_{e \in \mathcal{E}_{h}^{I} \cup \mathcal{E}_{h}^{D}} \int_{e}\left\{\frac{\partial u}{\partial \mathbf{n}}\right\} \llbracket \gamma_{h}\left(u_{I}-u_{h}\right) \rrbracket d s \\
= & \sum_{T \in \mathcal{T}_{h}} \int_{T}-\Delta u \gamma_{h}\left(u_{I}-u_{h}\right) d x+\sum_{e \in \mathcal{E}_{h}^{C}} \int_{e}\left\{\frac{\partial u}{\partial \mathbf{n}}\right\} \llbracket \llbracket \gamma_{h}\left(u_{I}-u_{h}\right) \rrbracket d s \\
= & \int_{\Omega} f \gamma_{h}\left(u_{I}-u_{h}\right) d x+\sum_{e \in \mathcal{E}_{h}^{C}} \int_{e}\left(\frac{\partial u}{\partial \mathbf{n}}\right) \gamma_{h}\left(u_{I}-u_{h}\right) d s .
\end{aligned}
$$


On the other hand, setting $v_{h}=u_{I}$ in (4) gives

$$
A\left(u_{h}, u_{I}-u_{h}\right) \geq \int_{\Omega} f \gamma_{h}\left(u_{I}-u_{h}\right) d x .
$$

Then, in view of (18) and (19), we infer that

$$
\begin{aligned}
\mathbb{D}_{2} & =A\left(u-u_{h}, u_{I}-u_{h}\right) \\
& \leq \sum_{e \in \mathcal{E}_{h}^{C}} \int_{e}\left(\frac{\partial u}{\partial \mathbf{n}}\right) \gamma_{h}\left(u_{I}-u_{h}\right) d s .
\end{aligned}
$$

Let $\Gamma_{C}^{0}=\left\{x \in \Gamma_{C}: u(x)=0\right\}$ and $\Gamma_{C}^{+}=\left\{x \in \Gamma_{C}: u(x)>0\right\}$, we then divide the set of edges on $\mathcal{E}_{h}^{C}$ into three non-overlapping parts, i.e., $\mathcal{E}_{h}^{C}=\Gamma_{h}^{0} \cup \Gamma_{h}^{+} \cup \Gamma_{h}^{-}$, with

$$
\begin{aligned}
& \Gamma_{h}^{0}=\left\{e \in \mathcal{E}_{h}^{C}: e \in \Gamma_{C}^{0}\right\}, \\
& \Gamma_{h}^{+}=\left\{e \in \mathcal{E}_{h}^{C}: e \in \Gamma_{C}^{+}\right\}, \\
& \Gamma_{h}^{-}=\left\{e \in \mathcal{E}_{h}^{C}: e \cap \Gamma_{C}^{0} \neq \emptyset, e \cap \Gamma_{C}^{+} \neq \emptyset\right\} .
\end{aligned}
$$

Observing that $u_{h} \geq 0$ on any $e \in \mathcal{E}_{h}^{C}$, direct computation yields $\gamma_{h} u_{h} \geq 0$ on any $e \in \mathcal{E}_{h}^{C}$, this together the fact that $\frac{\partial u}{\partial \mathbf{n}} \geq 0$ on $\Gamma_{C}$ implies that

$$
\begin{aligned}
\mathbb{D}_{2} \leq & \sum_{e \in \mathcal{E}_{h}^{C}} \int_{e}\left(\frac{\partial u}{\partial \mathbf{n}}\right) \gamma_{h}\left(u_{I}-u_{h}\right) d s \\
= & \sum_{e \in \Gamma_{h}^{0}} \int_{e}\left(\frac{\partial u}{\partial \mathbf{n}}\right) \gamma_{h}\left(u_{I}-u_{h}\right) d s+\sum_{e \in \Gamma_{h}^{+}} \int_{e}\left(\frac{\partial u}{\partial \mathbf{n}}\right) \gamma_{h}\left(u_{I}-u_{h}\right) d s \\
& +\sum_{e \in \Gamma_{h}^{-}} \int_{e}\left(\frac{\partial u}{\partial \mathbf{n}}\right) \gamma_{h}\left(u_{I}-u_{h}\right) d s \\
\leq & \sum_{e \in \Gamma_{h}^{0}} \int_{e}\left(\frac{\partial u}{\partial \mathbf{n}}\right) \gamma_{h}\left(u_{I}\right) d s+\sum_{e \in \Gamma_{h}^{+}} \int_{e}\left(\frac{\partial u}{\partial \mathbf{n}}\right) \gamma_{h}\left(u_{I}\right) d s+\sum_{e \in \Gamma_{h}^{-}} \int_{e}\left(\frac{\partial u}{\partial \mathbf{n}}\right) \gamma_{h}\left(u_{I}-u_{h}\right) d s .
\end{aligned}
$$

Since $u=0$ on any $e \in \Gamma_{h}^{0}$, we conclude that $u_{I}=0$, thus,

$$
\sum_{e \in \Gamma_{h}^{0}} \int_{e}\left(\frac{\partial u}{\partial \mathbf{n}}\right) \gamma_{h}\left(u_{I}\right) d s=0 .
$$

If $e \in \Gamma_{h}^{+}$, we have $u>0$. This together with $u \frac{\partial u}{\partial \mathbf{n}}=0$ implies that $\frac{\partial u}{\partial \mathbf{n}}=0$, we then have

$$
\sum_{e \in \Gamma_{h}^{+}} \int_{e}\left(\frac{\partial u}{\partial \mathbf{n}}\right) \gamma_{h}\left(u_{I}\right) d s=0 .
$$

Inserting (22) and (23) into (21) yields

$$
\begin{aligned}
\mathbb{D}_{2} \leq & \sum_{e \in \Gamma_{h}^{-}} \int_{e}\left(\frac{\partial u}{\partial \mathbf{n}}\right) \gamma_{h}\left(u_{I}-u_{h}\right) d s \\
= & {\left[\sum_{e \in \Gamma_{h}^{-}} \int_{e}\left(\frac{\partial u}{\partial \mathbf{n}}\right) \gamma_{h}\left(u_{I}-u_{h}\right) d s-\sum_{e \in \Gamma_{h}^{-}} \int_{e}\left(\frac{\partial u}{\partial \mathbf{n}}\right)\left(u_{I}-u_{h}\right) d s\right] } \\
& +\sum_{e \in \Gamma_{h}^{-}} \int_{e}\left(\frac{\partial u}{\partial \mathbf{n}}\right)\left(u_{I}-u_{h}\right) d s \\
\equiv & \mathbb{D}_{21}+\mathbb{D}_{22} .
\end{aligned}
$$


Set $\theta=u_{I}-u_{h}$, we rewrite $\mathbb{D}_{21}$ as

$$
\mathbb{D}_{21}=\sum_{e \in \Gamma_{h}^{-}} \int_{e}\left(\frac{\partial u}{\partial \mathbf{n}}\right)\left(\gamma_{h} \theta-\theta\right) d s .
$$

Combining (9), (13) and Cauchy-Schwarz inequality, we have, for any $e \in \Gamma_{h}^{-}$,

$$
\begin{aligned}
\int_{e}\left(\frac{\partial u}{\partial \mathbf{n}}\right)\left(\gamma_{h} \theta-\theta\right) d s & \leq\left\|\frac{\partial u}{\partial \mathbf{n}}\right\|_{L^{\infty}(e)} \int_{e}\left|\gamma_{h} \theta-\theta\right| d s \\
& \leq C_{1} h_{e}^{1 / 2}\left\|\frac{\partial u}{\partial \mathbf{n}}\right\|_{L^{\infty}(e)}\left\|\gamma_{h} \theta-\theta\right\|_{0, e} \\
& \leq C_{2} h\left\|\frac{\partial u}{\partial \mathbf{n}}\right\|_{L^{\infty}(e)}|\theta|_{1, T},
\end{aligned}
$$

where $e \in \mathcal{E}_{h}^{T}$. Therefore,

$$
\begin{aligned}
\mathbb{D}_{21} & =\sum_{e \in \Gamma_{h}^{-}} \int_{e}\left(\frac{\partial u}{\partial \mathbf{n}}\right)\left(\gamma_{h} \theta-\theta\right) d s \\
& \leq C_{2} h\left\|\frac{\partial u}{\partial \mathbf{n}}\right\|_{L^{\infty}\left(\Gamma_{C}\right)} \sum_{e \in \Gamma_{h}^{-}}|\theta|_{1, T} \\
& \leq C_{3} h\left\|\frac{\partial u}{\partial \mathbf{n}}\right\|_{L^{\infty}\left(\Gamma_{C}\right)}\|\theta\|_{h} \\
& \leq \frac{C_{3}}{4 \epsilon_{2}} h^{2}\left\|\frac{\partial u}{\partial \mathbf{n}}\right\|_{L^{\infty}\left(\Gamma_{C}\right)}^{2}+C_{3} \epsilon_{2}\left\|u_{I}-u_{h}\right\|_{h^{2}}^{2} .
\end{aligned}
$$

Here in the third line we have used the assumption that the number of transition points is finite.

We now turn to bound the term $\mathbb{D}_{22}$. For any $e \in \Gamma_{h}^{-}$, we infer from (10), (13) and Cauchy-Schwarz inequality that

$$
\begin{aligned}
\int_{e}\left(\frac{\partial u}{\partial \mathbf{n}}\right)\left(u_{I}-u\right) d s & \leq\left\|\frac{\partial u}{\partial \mathbf{n}}\right\|_{L^{\infty}(e)} \int_{e}\left|u_{I}-u\right| d s \\
& \leq C_{4} h_{e}^{1 / 2}\left\|\frac{\partial u}{\partial \mathbf{n}}\right\|_{L^{\infty}(e)}\left\|u-u_{I}\right\|_{0, e} \\
& \leq C_{5} h^{2}\left\|\frac{\partial u}{\partial \mathbf{n}}\right\|_{L^{\infty}(e)}|u|_{2, T},
\end{aligned}
$$

where $e \in \mathcal{E}_{h}^{T}$. Then it follows that

$$
\begin{aligned}
\mathbb{D}_{22} & =\sum_{e \in \Gamma_{h}^{-}} \int_{e}\left(\frac{\partial u}{\partial \mathbf{n}}\right)\left(u_{I}-u\right) d s \\
& \leq C_{5} h^{2}\left\|\frac{\partial u}{\partial \mathbf{n}}\right\|_{L^{\infty}\left(\Gamma_{C}\right)} \sum_{e \in \Gamma_{\bar{h}}^{-}}|u|_{2, T} \\
& \leq C_{6} h^{2}\left\|\frac{\partial u}{\partial \mathbf{n}}\right\|_{L^{\infty}\left(\Gamma_{C}\right)}|u|_{2} .
\end{aligned}
$$

Here we have used the assumption that the number of transition points is finite.

Combining (16), (17), (27) and (29), we arrive at

$$
\begin{aligned}
& \left(C_{b}-C_{a} \epsilon_{1}-C_{3} \epsilon_{2}\right)\left\|u_{I}-u_{h}\right\|_{h}^{2} \\
& \leq \frac{C_{a}}{4 \epsilon_{1}}\left\|u-u_{I}\right\|_{h}^{2}+\frac{C_{3}}{4 \epsilon_{2}} h^{2}\left\|\frac{\partial u}{\partial \mathbf{n}}\right\|_{L^{\infty}\left(\Gamma_{C}\right)}^{2}+C_{6} h^{2}\left\|\frac{\partial u}{\partial \mathbf{n}}\right\|_{L^{\infty}\left(\Gamma_{C}\right)}|u|_{2} .
\end{aligned}
$$

Choosing appropriate parameters $\epsilon_{i}(i=1,2)$ such that $C_{b}-C_{a} \epsilon_{1}-C_{3} \epsilon_{2}>0$, the desired estimate can be obtained by gathering estimates (12), (15) and (30). 


\section{Conclusion}

We proposed and analyzed a discontinuous finite volume method for the Signorini problem. Optimal order a priori error analysis in the energy norm is provided. In the future work, we shall mainly develop a posteriori error analysis.

\section{Acknowledgements}

This work was supported by Guangdong Basic and Applied Basic Research Foundation (Grant Nos. 2020A1515011032 and 2018A030307024).

\section{References}

Kinderlehrer, D., \& Stampacchia, G. (1980). An Introduction to Variational Inequalities and Their Applications. Academic Press, New York.

Falk, S. (1974). Error estimates for the approximation of a class of variational inequalities. Math. Comput., 28, 963-971. https://doi.org/10.1090/S0025-5718-1974-0391502-8

Brezzi, F., Hager, W. W., \& Raviart, P. A. (1977). Error estimates for the finite element solution of variational inequalities, Part I: primal theory. Numer. Math., 28, 431-443. https://doi.org/10.1007/BF01404345

Scarpini, F., \& Vivaldi, M. (1977). Error estimates for the approximation of some unilateral problems. RAIRO Anal. Numer., 11, 197-208. https://doi.org/10.1051/m2an/1977110201971

Glowinski, R. (2008). Numerical Methods for Nonlinear Variational Problems. Springer-Verlag, Berlin.

Ben Belgacem, F. (2000). Numerical simulation of some variational inequalities arisen from unilateral contact problems by the finite element method. SIAM J. Numer. Anal., 37, 1198-1216. https://doi.org/10.1137/S0036142998347966

Chen, Z., \& Nochetto, R. (2000). Residual type a posteriori error estimates for elliptic obstacle problems. Numer. Math., 84, 527-548. https://doi.org/10.1007/s002110050009

Veeser, A. (2001). Efficient and reliable a posteriori error estimators for elliptic obstacle problems. SIAM J. Numer. Anal., 39, 146-167. https://doi.org/10.1137/S0036142900370812

Hild, P., \& Nicaise, S. (2005). A posteriori error estimations of residual type for Signorini's problem. Numer. Math., 101, 523-549. https://doi.org/10.1007/s00211-005-0630-5

Carstensen, C., \& Hu, J. (2015). An optimal adaptive finite element method for an obstacle problem. Comput. Methods Appl. Math., 15, 259-277. https://doi.org/10.1515/cmam-2015-0017

Wang, L. (2003). On the error estimate of nonconforming finite element approximation to the obstacle problem. $J$. Comput. Math., 21, 481-490. http://www.global-sci.org/intro/article_detail/jcm/10251.html

Hua, D., \& Wang, L. (2007). The nonconforming finite element method for Signorini problem. J. Comput. Math., 25, 67-80. http://www.global-sci.org/intro/article_detail/jcm/8673.html

Shi, D., Ren, J., \& Gong, W. (2012). Convergence and superconvergence analysis of a nonconforming finite element method for solving the Signorini problem. Nonlinear Anal. RWA., 75, 3493-3502. https://doi.org/10.1016/j.na.2012.01.007

Shi, D., \& Xu, C. (2013). $E Q_{1}^{\text {rot }}$ nonconforming finite element approximation to Signorini problem. Sci. China Math., 56, 1301-1311. https://doi.org/10.1007/s11425-013-4615-z

Carstensen, C., \& Köhler, K. (2017). Nonconforming FEM for the obstacle problem. IMA J. Numer. Anal., 37, 64-93. https://doi.org/10.1093/imanum/drw005

Arnold, D. N., Brezzi, F., Cockburn, B., \& Marini, L. D. (2002). Unified analysis of discontinuous Galerkin methods for elliptic problems. SIAM J. Numer. Anal., 39, 1749-1779. https://doi.org/10.1137/S0036142901384162

Djoko, J. K. (2008). Discontinuous Galerkin finite element methods for variational inequalities of first and second kinds. Numer. Methods PDEs., 24, 296-311. https://doi.org/10.1002/num.20261

Wang, F., Han, W., \& Cheng, X. (2010). Discontinuous Galerkin methods for solving elliptic variational inequalities. SIAM J. Numer. Anal., 48, 708-733. https://doi.org/10.1137/09075891X

Wang, F., Han, W., \& Cheng, X. (2011). Discontinuous Galerkin methods for solving the Signorini problem. IMA. J. Numer. Anal., 31, 1754-1772. https://doi.org/10.1093/imanum/drr010

Bustinza, R., \& Sayas, F. J. (2012). Error estimates for an LDG method applied to Signorini type problems. J. Sci. Comput., 52, 322-339. https://doi.org/10.1007/s10915-011-9548-5 
Gudi, T., \& Kamana, P. (2014). A posteriori error control of discontinuous Galerkin methods for elliptic obstacle problems. Math. Comput., 83, 579-602. https://doi.org/10.1090/S0025-5718-2013-02728-7

Gudi, T., \& Kamana, P. (2016). A posteriori error estimates of discontinuous Galerkin methods for the Signorini problem. J. Comput. Appl. Math., 292, 257-278. https://doi.org/10.1016/j.cam.2015.07.008

Zeng, Y., Chen, J., \& Wang, F. (2015). Error estimates of the weakly over-penalized symmetric interior penalty method for two variational inequalities. Comput. Math. Appl., 69, 760-770. https://doi.org/10.1016/j.camwa.2015.02.022

Eymard, R., Gallouët, T., \& Herbin, R. (2000). Finite Volume Methods: Handbook of Numerical Analysis. North-Holland, Amsterdam.

Li, R., Chen, Z., \& Wu, W. (2000). Generalized Difference Methods for Differential Equations: Numerical Analysis of Finite Volume Methods. Marcel Dekker, New York.

Cai, Z., Mandel, J., \& McCormick, S. (1991). The finite volume element method for diffusion equations on general triangulations. SIAM J. Numer. Anal., 28, 392-402. https://doi.org/10.1137/0728022

Huang, J., \& Xi, S. (1998). On the finite volume element method for general self-adjoint elliptic problems. SIAM J. Numer. Anal., 35, 1762-1774. https://doi.org/10.1137/S0036142994264699

Ewing, R. E., Lin, T., \& Lin, Y. (2002). On the accuracy of the finite volume element method based on piecewise linear polynomials. SIAM J. Numer. Anal., 39, 1865-1888. https://doi.org/10.1137/S0036142900368873

Wu, H., \& Li, R. (2003). Error estimates for finite volume element methods for general second-order elliptic problems. Numer. Methods PDEs., 19, 693-708. https://doi.org/10.1002/num.10068

Chou, S. H., \& Ye, X. (2007). Unified analysis of finite volume methods for second order elliptic problems. SIAM J. Numer. Anal., 45, 1639-1653. https://doi.org/10.1137/050643994

Xu, J., \& Zou, Q. (2009). Analysis of linear and quadratic simplicial finite volume methods for elliptic equations. Numer. Math., 111, 469-492. https://doi.org/10.1007/s00211-008-0189-z

Chen, L. (2010). A new class of high order finite volume methods for second order elliptic equations. SIAM J. Numer. Anal., 47, 4021-4043. https://doi.org/10.1137/080720164

Yang, M., \& Liu, J. (2011). A quadratic finite volume element method for parabolic problems on quadrilateral meshes. IMA J. Numer. Anal., 31, 1038-1061. https://doi.org/10.1093/imanum/drp054

Lv, J., \& Li, Y. (2015). Optimal biquadratic finite volume element methods on quadrilateral meshes. SIAM J. Numer. Anal., 50, 2379-2399. https://doi.org/10.1137/100805881

Zhang, Z., \& Zou, Q. (2015). Vertex centered finite volume schemes of any order over quadrilateral meshes for elliptic boundary problems. Numer. Math., 130, 363-393. https://doi.org/10.1007/s00211-014-0664-7

Guo, L., Li, H., \& Zou, Q. (2019). Interior estimate of finite volume element methods over quadrilateral meshes for elliptic equations. SIAM J. Numer. Anal., 57, 2246-2265. https://doi.org/10.1137/18M1197746

Zhang, Y., Yang, M., \& Chen, C. (2019). The hybrid Wilson finite volume method for elliptic problems on quadrilateral meshes. Adv. Comput. Math., 45, 429-452. https://doi.org/10.1007/s10444-018-9623-7

Zhang, T., \& Tang, L. (2015). Finite volume method for the variational inequalities of first and second kinds. Math. Methods Appl. Sci., 38, 3980-3989. https://doi.org/10.1002/mma.3331

Zhang, T., \& Li, Z. (2015). An analysis of finite volume element method for solving the Signorini problem. Appl. Math. Comput., 270, 830-841. https://doi.org/10.1016/j.amc.2015.08.106

Ye, X. (2004). A new discontinuous finite volume method for elliptic problems. SIAM J. Numer. Anal., 42, 1062-1072. https://doi.org/10.1137/S0036142902417042

Kumar, S., Nataraj, N., \& Pani, A. K. (2009). Discontinuous Galerkin finite volume element methods for second-order linear elliptic problems. Numer. Methods PDEs., 25, 1402-1424. https://doi.org/10.1002/num.20405

Liu, J., Mu, L., Ye, X., \& Jari, R. (2012). Convergence of the discontinuous finite volume method for elliptic problems with minimal regularity. J. Comput. Appl. Math., 236, 4537-4546. https://doi.org/10.1016/j.cam.2012.05.009

Bi, C., \& Geng, J. (2012). A discontinuous finite volume element method for second-order elliptic problems. Numer. Methods PDEs., 28, 425-440. https://doi.org/10.1002/num.20626

Carstensen, C., Nataraj, N., \& Pani, A. K. (2016). Comparison results and unified analysis for first-order finite volume 
element methods for a Poisson model problem. IMA. J. Numer. Anal., 36, 1120-1142. https://doi.org/10.1093/imanum/drv050

Ye, X. (2006). A discontinuous finite volume method for the Stokes problems. SIAM J. Numer. Anal., 44, 183-198. https://doi.org/10.1137/040616759

Cui, M., \& Ye. X. (2010). Unified analysis of finite volume methods for the Stokes equations. SIAM J. Numer. Anal., 48, 824-839. https://doi.org/10.1137/090780985

Kumar, S., \& Ruiz-Baier, R. (2015). Equal order discontinuous finite volume element methods for the Stokes problem. $J$. Sci Comput., 65, 956-978. https://doi.org/10.1007/s10915-015-9993-7

Wang, J., Wang, Y., \& Ye, X. (2018). A unified a posteriori error estimator for finite volume methods for the Stokes equations. Math. Methods Appl. Sci., 41, 866-880. https://doi.org/10.1002/mma.2871

Carstensen, C., Dond, K., Nataraj, N., \& Pani, A. K. (2018). Three First-order finite volume element methods for Stokes equations under minimal regularity assumptions. SIAM J. Numer. Anal., 56, 2648-2671. https://doi.org/10.1137/17M1134135

Wang, G., He, Y., \& Li. R. (2016). Discontinuous finite volume methods for the stationary Stokes-Darcy problem. Int. J. Numer. Meth. Engrg., 107, 395-418. https://doi.org/10.1002/nme.5171

Li, R., Gao, Y., Li, J., \& Chen, Z. (2018). Discontinuous finite volume element method for a coupled nonstationary Stokes-Darcy problem. J. Sci. Comput., 74, 693-727. https://doi.org/10.1007/s10915-017-0454-3

Kumar, S., Oyarzúa, R., Ruiz-Baier, R., \& Sandilya, R. (2020). Conservative discontinuous finite volume and mixed schemes for a new four-field formulation in poroelasticity. ESAIM: M2AN., 54, 273-299. https://doi.org/10.1051/m2an/2019063

Li, R., Gao, Y., Chen, J., Zhang, L., He, X., \& Chen, Z. (2020). Discontinuous finite volume element methodfor a coupled Navier-Stokes-Cahn-Hilliard phase field model. Adv. Comput. Math., 46, 1-35. https://doi.org/10.1007/s10444-020-09764-4

Bürger, B., Kumar, S., \& Ruiz-Baier, R. (2015). Discontinuous finite volume element discretization for coupled flowtransport problems arising in models of sedimentation. J. Comput. Phys., 299, 446-471. https://doi.org/10.1016/j.jcp.2015.07.020

Kumar, S., Ruiz-Baier, E., \& Sandilya, R. (2019). Error bounds for discontinuous finite volume discretisations of Brinkman optimal control problems. J. Sci. Comput., 78, 64-93. https://doi.org/10.1007/s10915-018-0749-z

Brenner, S. C., \& Scott, L. R. (2008). The Mathematical Theory of Finite Element Methods (3rd ed.). Springer-Verlag, New York.

Wang, M., \& Shi, Z. (2010). Finite Element Methods. Beijing: Science Press.

\section{Copyrights}

Copyright for this article is retained by the author(s), with first publication rights granted to the journal.

This is an open-access article distributed under the terms and conditions of the Creative Commons Attribution license (http://creativecommons.org/licenses/by/4.0/). 